\title{
Continuity in transition from paediatric to adult healthcare
}

\author{
AR Watson \\ Emeritus Professor, Children's Renal \& Urology Unit, Nottingham University Hospitals NHS Trust, Nottingham Children's Hospital, \\ Nottingham, UK
}

KEYWORDS Transition, adolescence, youth work

Correspondence to AR Watson

DECLARATION OF INTERESTS No conflicts of interest declared.

\author{
Children's Renal \& Urology Unit \\ Nottingham University Hospitals \\ NHS Trust, Nottingham Children's \\ Hospital, QMC Campus, \\ Derby Road, Nottingham \\ NG7 2UH, UK
}

tel. +44 (0) II 9709420

e-mail judith.hayes@nuh.nhs.uk
Attention is being focused on the transition from paediatric to adult care as the number of young people with chronic illness or disability increases. ${ }^{1-3}$ Children and young people with conditions such as cystic fibrosis, diabetes, chronic renal failure, congenital heart disease and cancer have been under the care of multidisciplinary teams who not only helped to manage their chronic illness but have also given attention to their psychological, social and educational development. The transition process raises a number of issues for these patients. The 'culture' of support is often different in adult centres where the patient is assumed to be an autonomous individual who should be able to make decisions for him or herself and have all the requisite self-management skills. Brain development in young people however may not be complete until patients are in their mid-20s and this can be related to increased risk-taking and impulsivity issues. ${ }^{4}$ Transfer to the adult unit may also take place at a time when adherence to therapy is at its most problematic.

Transition to adult care needs to be a planned process, occurring over many years. Whatever the age of the individual, there should be support on the adult side as the 'transition' to adulthood continues. Consensus statements have been published on the generic transition issues widely applicable to young people moving from paediatric to adult care, including an extensive document by the Royal College of Physicians of Edinburgh., ${ }^{5,6}$ As transition issues induce anxiety in patient, carers and staff, it is important that the viewpoints of all involved are sought. However the logistics of when, where and how to transition need to be resolved for each subspecialty programme or group. There might, for example, be a large enough cohort of diabetic patients locally to establish a viable transition clinic between paediatric and adult clinics, where young people are seen by both paediatric and adult diabetologists. However, with chronic kidney disease young people may be transferring from a regional or supraregional centre to a number of distant adult units in different cities and therefore only single transfer clinics are available.
Joint paediatric and adult consensus statements can establish important standards for transition. Such agreement has been achieved recently by the International Society of Nephrology and the International Pediatric Nephrology Association. ${ }^{7}$ The guideline emphasises that young people should be:

- introduced to the concept of transition in early adolescence (12-14 years old).

- given information about transition in a gradual manner, appropriate to developmental stage and intellectual ability.

- have access to identified lead clinicians (transition champions) in both paediatric and adult units to coordinate and educate on transition issues.

- assigned to a nominated key worker who can assist in the transition process.

- provided with a generic transition plan which can then be individualised for each patient.

- reassured that parents, other family members and boyfriends/girlfriends (if the young person agrees) can be included. More information lessens anxieties.

- offered the opportunity of an informal visit to the nominated adult service before transfer occurs.

- given the opportunity to participate in group sessions with other young people who are about to transition.

- $\quad$ offered peer support experience. Peer support can be complimented by establishing local email and social networking groups.

- able to receive tools to aid in the acquisition of disease self-management skills such as a transition medical passport and transition scale.

The transfer process from the paediatric to adult unit needs to be individualised and agreed with each patient and his/her family/carers in conjunction with the paediatric and adult teams. It should take place during a period without crisis and after completing school education (usually age 18 in the UK). The plan should take into account other subspecialties involved with the 
young person's care. Transfer must also take place with due consideration to financial factors and not be done abruptly as a result of financial pressures, without adequate preparation.

This consensus statement has given fresh impetus to the concept of transition champions, clinicians on both sides who have taken a special interest in transition and can provide the guiding hands across any divide between paediatric and adult units. The development of specialist nursing roles has also provided an opportunity for liaison. We know that young people often relate best to their own peers and our own unit has benefited greatly from youth workers who have encouraged the peer support and peer mentorship schemes. ${ }^{8}$ Many young people now attend a transition residential session over a weekend or a few days outside the hospital where coaching about transition can be delivered by those who have themselves undergone the process. ' Some adult units are now evaluating similar young adult support and young adult clinics.

\section{ARE TRANSITIONAL CARE PROGRAMMES EFFECTIVE?}

A recent systematic review of studies that consistently evaluated health outcomes following transition programmes, either by comparison with a control group or by measurement of pre-intervention or postintervention outcomes, reported only ten studies that met the inclusion criteria and most were in diabetes. ${ }^{10}$ Six of these showed statistically significant improvements in outcomes. Existing evidence supports patient education

\section{REFERENCES}

I Watson AR. Problems and pitfalls of transition from paediatric to adult renal care. Pediatr Nephrol 2005; 20:1/3-7. http://dx.doi. org/I0.1007/s00467-004-1763-y

2 Viner RM. Transition of care from pediatric to adult services: one part of improved health services for adolescents. Arch Dis Child 2008; 98:160-3. http://dx.doi.org//0.1/36/adc.2006.103721

3 Betz CL. Approaches to transition in other chronic illnesses and conditions. Pediatr Clin North Am 2010; 57:983-96. http://dx.doi org/I0.1016/j.pcl.2010.07.017

4 Giedd JN.The teen brain: insights from neuroimaging.J Adolesc Health 2008; 42:335-43. http://dx.doi.org//0.1016/j.jadohealth.2008.01.007

5 Webb N, Harden P, Lewis $\mathrm{C}$ et al. Building consensus on transition of transplant patients from pediatric to adult healthcare. Arch Dis Child 2010; 95:606-I I. http://dx.doi.org/I 0.I I 36/adc.2009.176255

6 The Royal College of Physicians of Edinburgh Transitions Steering Group. Think transition: developing the essential link between paediatric and adult care [Internet]. Edinburgh: Royal College of Physicians of Edinburgh; 2008 [cited 20II Dec 21]. Available at: http://www.rcpe.ac.uk/clinical-standards/documents/transition.pdf and specific transition clinics (either jointly staffed by paediatric and adult physicians or dedicated young adult clinics within adult services). Obviously a lot more needs to be done to evaluate programmes within different contexts, but globally the problem is still finding time and funding to establish transition/transfer clinics. Economically, as well as personally, transition support may provide good value. A recent report, for example, compared renal transplant patients who had been transferred following a transition programme with a retrospective cohort who had received no transition support. The costs were less for those who had undergone transition planning but, more importantly, none of these patients died or lost their grafts, compared with $24 \%$ of those who had received no transition preparation. Costs were considerably higher for those patients who had lost their transplants and returned to dialysis.

\section{CONCLUSIONS}

To provide essential continuity of care every paediatric centre should have a transition programme, adapted by each subspecialty, which effectively engages patients, carers, paediatric and adult staff in the process.

Transfer to adult care should only take place after the young person has undergone the majority of a transition process that continues in the adult unit. The momentum to develop transition services will be increased with jointly developed national or international consensus statements, against which standards can be audited and research conducted.
7 Watson AR, Harden PN, Ferris ME et al. Transition from pediatric to adult renal services: a consensus statement by the International Society of Nephrology (ISN) and the International Pediatric Nephrology Association (IPNA).Kidney Int 20I I;80:704-7.http://dx.doi.org/I0.1038/ ki.20II.209

8 Watson AR. Hospital youth work and adolescent support. Arch Dis Child 2004; 89:440-2. http://dx.doi.org// 0.I I36/adc.2002.022855

9 Watson AR, Hilton D, Hackett D. Therapeutic recreation camps to provide a residential experience for young people in transition to adult renal units. Pediatr Nephrol 2010; 25:787-8. http://dx.doi.org//0.1007/ s00467-009-I367-7

10 Crowley R,Wolfe I, Lock K et al. Improving the transition between paediatric and adult healthcare: a systematic review. Arch Dis Child 20I I; 96:548-53. http://dx.doi.org/I0.I I36/adc.20I0.202473 